\section{Internalized chitosan nanoparticles persist for long time in cultured cells}

\author{
M. Malatesta, ${ }^{1}$ S. Grecchi, ${ }^{1}$ E. Chiesa, ${ }^{2}$ \\ B. Cisterna, ${ }^{1}$ M. Costanzo, ${ }^{1}$ C. Zancanaro ${ }^{1}$ \\ 'Department of Neurological and \\ Movement Sciences, Anatomy and \\ Histology Section, University of Verona \\ 2Department of Drugs Sciences, \\ University of Pavia, Italy
}

\section{Abstract}

Chitosan-based nanoparticles (chiNPs) are considered to be potentially good carriers for the sustained intracellular delivery of specific molecules. However, scarce attention has been paid to the long-lasting permanence of these NPs in the intracellular milieu, as well as to their intracellular fate (i.e., distribution, interaction with cell organelles, and degradation) in the long term. In the present study, the presence and subcellular location of FITC-labelled chiNPs were monitored in HeLa cells up to 14 days post-administration using multicolorfluorescence confocal microscopy and diaminobenzidine photo-oxidation at transmission electron microscopy. The main result of the present study is the demonstration that internalized chiNPs persist inside the cell up to two weeks, occurring in both the cytoplasm and nucleus; accordingly, chiNPs are able to pass from mother to daughter cells through several mitotic cycles. The cells did not show increased mortality or structural damage up to 14 days after chiNP exposure.

\section{Introduction}

Chitosan is a unique natural cationic polysaccharide exhibiting peculiar characteristics of wide availability, sustainability, functionalizability, biocompatibility and biodegradability. ${ }^{1}$ In particular, the presence of active amino groups on the polymeric backbone makes polymer preparation simple and mild, while allowing chitosan to be easily modified chemically. Based on these features, chitosan has become one of the most popular biopolymers for the development of drug delivery systems (recent reviews in ${ }^{2-4}$ ).

Diverse chitosan-based nanoparticles (chiNPs) have been successfully used to deliver specific molecules inside cells and tissues, thanks to their physicochemical properties promoting the interaction with the negatively charged domains of the cell membrane ${ }^{5,6}$ and enhancing the endocytotic uptake. ${ }^{7,8}$ At the same time, these properties allow chiNP escape from the endosomes, thus limiting lysosomal degradation, ${ }^{9-12}$ and support the gradual release of the encapsulated molecules to the target site. ${ }^{10,13-16}$ ChiNPs are therefore considered to be potentially good carriers for the sustained intracellular delivery of specific molecules, but scarce attention has been paid to the long-lasting permanence of these NPs in the intracellular milieu, as well as to their intracellular fate (i.e., distribution, interaction with cell organelles, and degradation) in the long (weeks) term. It has been previously demonstrated that chiNPs effectively enter human epithelial cells in culture ${ }^{15}$ and gradually release the encapsulated drug into the intracellular milieu. ${ }^{15,16}$

In the present study, the presence and subcellular location of FITC-labelled chiNPs were monitored in HeLa cells up to 14 days postadministration using multicolor-fluorescence confocal microscopy and diaminobenzidine photo-oxidation at transmission electron microscopy.

\section{Materials and Methods}

\section{Preparation of chiNPs}

Chitosan chloridrate (Protasan CL113, Mw $150,000 \mathrm{Da}$, degree of acetylation 17\%, was obtained from Pronova Biomedical (Oslo, Norway). Fluorescein 5(6)-isothiocyanate (FITC, Mw 398.38) and tripolyphosphate pentasodium salt (TPP, Mw 367.9 Da) were purchased from Sigma-Aldrich Chemical Company (Gillingham, UK).

FITC-labelled chitosan was synthetized by adding $2.5 \mathrm{~mL}$ of FITC in methanol $(2 \mathrm{mg} / \mathrm{mL})$ to $5 \mathrm{~mL}$ of chitosan $(1 \% \mathrm{w} / \mathrm{v}$ in $0.1 \mathrm{~N}$ $\mathrm{CH}_{3} \mathrm{COOH}$ :methanol 1:1 v/v). The reaction was run for $3 \mathrm{~h}$ in the dark at room temperature. Then the labelled polymer was precipitated in $0.5 \mathrm{M} \mathrm{NaOH}$ (till to $\mathrm{pH} 10$ ). The precipitate was recovered by centrifugation at $16,400 \mathrm{rpm}$ (10 min) at $4^{\circ} \mathrm{C}$ and washed in methanol:water $(70: 30 \mathrm{v} / \mathrm{v})$. The washing and the pelletization were repeated until no fluorescence was detected in the supernatant (Luminescence Spectrophotometer LS55, Perkin Elmer, Waltham, MA, USA). The labelled chitosan was then dissolved in $0.1 \mathrm{~N} \mathrm{CH}_{3} \mathrm{COOH}$ and dialyzed in the dark against distilled water for 3 days. Finally, the FITC-labelled chitosan was freezedried. The labelling efficiency (\% FITC/FITClabelled chitosan, w/w) was determined by measuring the fluorescence intensity of the FITC-labelled chitosan solution against standard solution of FITC (Luminescence
Correspondence: Manuela Malatesta, Department of Neurological and Movement Sciences, Anatomy and Histology Section, University of Verona, Strada Le Grazie 8, 37134 Verona, Italy.

Tel. +39.045.8027155 - Fax: 39.045.8027163.

E-mail:manuela.malatesta@univr.it

Keywords: Nanoparticles, endosomal escape, cell nucleus, DAB photo-oxidation.

Acknowledgments: the authors are grateful for useful advice and suggestions from Prof. B. Conti and Prof. I. Genta. Confocal fluorescence micrographs were taken at the Centro Grandi Strumenti of the University of Pavia (http://cgs.unipv.it). This work and B.C. fellowship were supported by Fondazione Cariverona, project Verona Nanomedicine Initiative. E.C. and M.C. are PhD students in receipt of fellowships from Doctoral Programs of the University of Pavia and Verona, respectively.

Received for publication: 28 January 2015.

Accepted for publication: 4 February 2015.

This work is licensed under a Creative Commons Attribution NonCommercial 3.0 License (CC BYNC 3.0).

(C) Copyright M. Malatesta et al., 2015

Licensee PAGEPress, Italy

European Journal of Histochemistry 2015; 59:2492 doi:10.4081/ejh.2015.2492

Spectrometer LS55, Perkin-Elmer). Fluorescent chiNPs were prepared with FITClabelled chitosan $\left(1 \mathrm{mg} / \mathrm{mL}\right.$ in $\left.0.1 \mathrm{~N} \mathrm{CH}_{3} \mathrm{COOH}\right)$ by a modified method combining ionotropic gelation and ultrasonication. ${ }^{17,18}$ Briefly, $2 \mathrm{~mL}$ of TPP aqueous solution $(0.75 \mathrm{mg} / \mathrm{mL})$ was added to $10 \mathrm{~mL}$ of $1 \mathrm{mg} / \mathrm{mL}$ chitosan chloridrate in acetic solution $(0.1 \mathrm{M})$ at the constant rate of $0.5 \mathrm{~mL} / \mathrm{min}$ under magnetic stirrer (Heidolph MR 3001, Heidolph Instruments GmbH \& Co., Germany) at 700 rpm while ultrasonicated in a ultrasonic bath operating at $50 / 60 \mathrm{~Hz}$. NPs were recovered by ultracentrifugation at $4^{\circ} \mathrm{C}$ at $20,000 \mathrm{rpm}$ for $60 \mathrm{~min}$ (ultracentrifuge LS-75 with rotor type $60 \mathrm{Ti}$, Beckman Coulter, Brea, CA, USA) and resuspended in $500 \mu \mathrm{L}$ of double-distilled water. Diameter, polydispersity and Zeta-potential (a parameter related to NP stability in a dispersion) of FITC-labelled chiNPs were determined by dynamic light scattering using a NICOMP 380 ZLS apparatus (Particle Sizing Systems, Menlo Park, CA, USA). Samples were diluted with $10 \mathrm{mM} \mathrm{NaCl}$ aqueous solution before measuring the particle size; sample run time was approximately $15 \mathrm{~min}$ (1 min for Zetapotential determination). All measurements were made in triplicate and mean values \pm SD were recorded. 


\section{In vitro cell culture}

HeLa cells $\left(5 \times 10^{4}\right)$ were grown in DMEM (Dulbecco Modified Eagles Medium) supplemented with $10 \%$ (v/v) fetal calf serum, $1 \%$ (w/v) glutamine, $100 \mathrm{U}$ of penicillin and 100 $\mu \mathrm{g} / \mathrm{mL}$ streptomycin (Celbio, Milan, Italy), at $37^{\circ} \mathrm{C}$ in a $5 \% \mathrm{CO}_{2}$ humidified atmosphere. Cells were trypsinized when subconfluent, and seeded on glass coverslips in 6 multiwell dishes for fluorescence and transmission electron microscopy. Two days after seeding, the initial medium was replaced with $1800 \mu \mathrm{L}$ of fresh medium plus $200 \mu \mathrm{L}$ of fluorescent chiNP suspension. Cells were kept in the medium containing chiNPs for $24 \mathrm{~h}$, and then this medium was replaced with fresh medium without chiNPs (retrieval). After retrieval, the cells were further grown for $24 \mathrm{~h}, 48 \mathrm{~h}, 72 \mathrm{~h}, 7$ days and 14 days. For long-term (i.e., $>72 \mathrm{~h}$ ) culture, the cells were originally seeded and exposed to chiNPs on flasks, let to grow in fresh medium therein, and finally detached and planted onto glass coverslips to be processed for fluorescence or electron microscopy.

\section{Cell viability assay}

To estimate the effect of chiNPs on cell viability, HeLa cultures at all the retrieval times were detached by mild trypsinization $(0.25 \%$ trypsin in PBS containing 0.05\% EDTA) and stained in suspension for 2 min with $0.1 \%$ Trypan blue in the culture medium: cells that were permeable to Trypan blue were considered as non-viable and their percentage was estimated by microscope counting on a Burker hemocytometer; cell samples not exposed to chiNPs were considered as controls. Results were expressed as the mean $\pm \mathrm{SE}$ of three independent experiments.

\section{Analysis of long-term chiNP intra- cellular distribution}

At each retrieval time, HeLa cells were fixed for fluorescence microscopy with $4 \%(\mathrm{v} / \mathrm{v})$ paraformaldehyde in PBS, pH 7.4 (30 min at room temperature, RT). The samples were stained for DNA with Hoechst $33258(1 \mu \mathrm{g} / \mathrm{mL}$ in PBS for $5 \mathrm{~min}$; Sigma-Aldrich, Buchs, Switzerland), counterstained with $0.1 \%$ Trypan blue in PBS for 2 min, rinsed in PBS, and mounted in a 1:1 mixture of glycerol:PBS (Calbiochem, Inalco, Milan, Italy): this allowed to visualize the intracellular presence of fluorescent chiNPs and to verify their possible intranuclear location. To elucidate whether internalized chiNPs undergo degradation in lysosomes, some cell samples were immunolabelled with a human autoimmune serum recognizing lysosomal proteins ${ }^{19}$ diluted 1:500 in PBS. After 60 min incubation at RT, immunolabelling was revealed with an Alexa 594-conjugated anti-human IgG antibody (Molecular Probes, Invitrogen, Milan, Italy) diluted 1:200 in PBS. The DNA was finally counterstained with 0.1 M Hoechst 33258 in PBS for 5 min, and cells were mounted in a 1:1 mixture of glycerol:PBS as above.

An Olympus BX51 microscope equipped with a 100W mercury lamp (Olympus Italia Srl, Milan, Italy) was used under the following conditions: $450-480 \mathrm{~nm}$ excitation filter (excf), $500 \mathrm{~nm}$ dichroic mirror (dm), and $515 \mathrm{~nm}$ barrier filter (bf) for FITC; $540 \mathrm{~nm}$ excf, $580 \mathrm{~nm}$ $\mathrm{dm}$, and $620 \mathrm{~nm}$ bf Alexa 594; 330-385 nm excf, $400-\mathrm{nm}$ dm, and $420 \mathrm{~nm}$ bf, for Hoechst 33258 . Images were recorded with an Olympus Magnifire digital camera system (Olympus Italia Srl), and stored on a PC by the Olympus software for processing and printing. For confocal laser scanning microscopy, a Leica TCSSP system mounted on a Leica DMIRBE inverted microscope (Leica Microsystems Italia, Milan, Italy) was used; for fluorescence excitation, an Ar/Vis laser at $488 \mathrm{~nm}$ for FITC, an $\mathrm{He} / \mathrm{Ne}$ laser at $543 \mathrm{~nm}$ for Alexa 594, and an Ar/UV laser at $364 \mathrm{~nm}$ for Hoechst 33258 were used. Spaced $(0.5 \mu \mathrm{m})$ optical sections were recorded using a $63 \mathrm{x}$ oil immersion objective. Images were collected in the $1024 \times 1024$ pixel format, stored on a magnetic mass memory

and processed by the Leica confocal software. Quantitative evaluation of chiNPs uptake was carried out by using an Olympus BX51 microscope: for each retrieval time, 1500 cells per sample were counted on several fields using a $40 \mathrm{x}$ objective lens and the mean percentage \pm SE of cells containing at least one chiNP was calculated. For transmission electron microscopy, HeLa cells were fixed with $2.5 \%$ (v/v) glutaraldehyde and 2\% (v/v) paraformaldehyde in $0.1 \mathrm{M}$ phosphate buffer, $\mathrm{pH} 7.4$, at $4^{\circ} \mathrm{C}$ for $1 \mathrm{~h}$, washed and incubated with 3,3 ' diaminobenzidine (DAB) $(20 \mathrm{mg} / 10 \mathrm{~mL}$ in Tris $\mathrm{HCl} 0.05 \mathrm{M}, \mathrm{pH}$ 7.6) under simultaneous irradiation with two 8W 0sram Blacklite 350 lamps for $2 \mathrm{~h}$ at room temperature (these lamps emit with high intensity in the spectral range between 430 and $470 \mathrm{~nm}$, thus being suitable for FITC excitation); the cells were then postfixed with $1 \% \mathrm{OsO}_{4}$ and $1.5 \%$ potassium ferrocyanide at room temperature for $1 \mathrm{~h}$, dehydrated with acetone and embedded in Epon. Samples processed as described above, but omitting either DAB incubation or exposure to the excitation light was used as control. Ultrathin sections were weakly stained with uranyl acetate and observed in a Philips

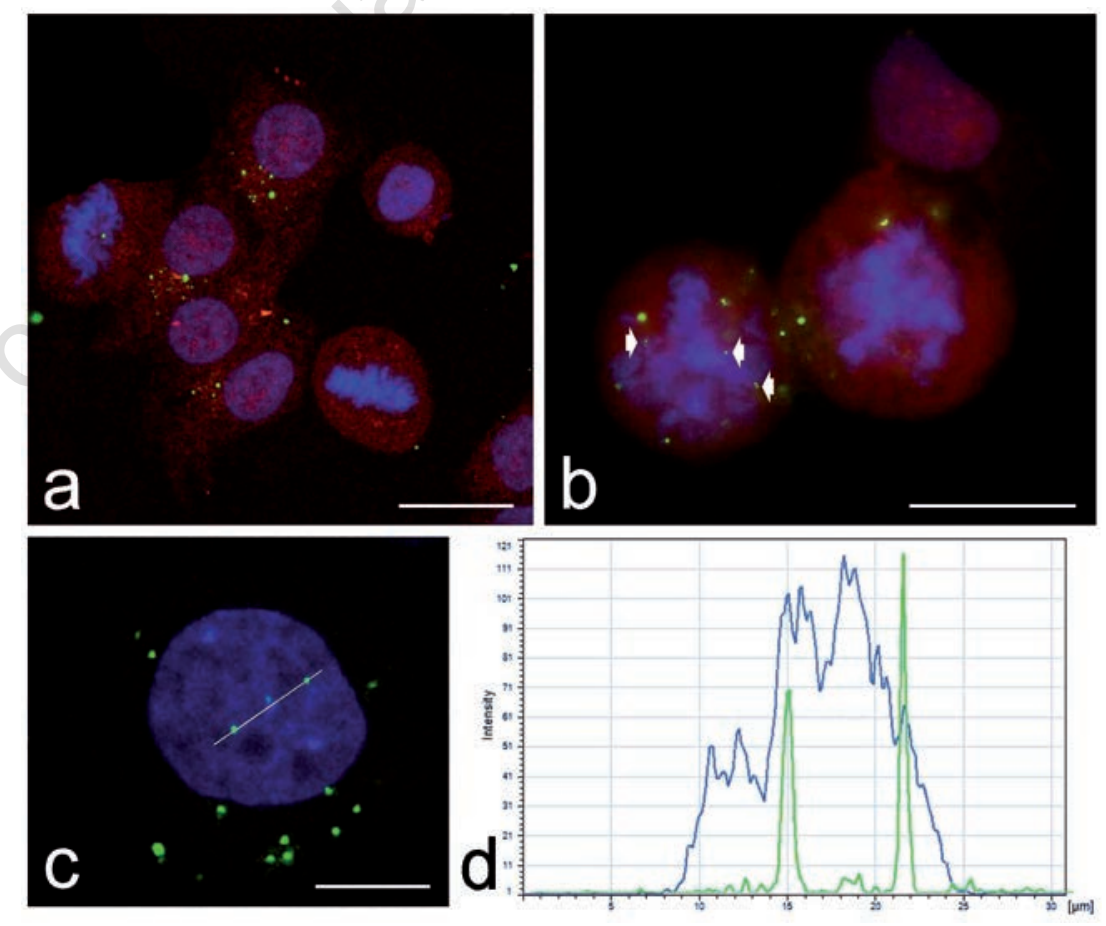

Figure 1. a,b) Confocal micrographs of HeLa cells $24 \mathrm{~h}$ after administration of FITClabelled chiNPs (green fluorescence): chiNPs may be observed in both interphasic and mitotic cells, where they may localize very close to the chromosome mass (arrowheads). The cells were mildly fixed with formaldehyde and counterstained with Trypan blue (red fluorescence); DNA was stained with Hoechst 33258 (blue fluorescence); scale bars: 50 $\mu \mathrm{m}$. c) Confocal optical section of an interphase nucleus containing two chiNPs ( $48 \mathrm{~h}$ after chiNP retrieval from the culture medium); scale bar: $20 \mu \mathrm{m}$. d) The colocalization of the green (chiNPs) and blue (DNA) signals was confirmed by histogram analysis of the fluorescence intensities along the white line in c). 
Morgagni transmission electron microscope (FEI Company Italia Srl, Milan, Italy) operating at $80 \mathrm{kV}$ and equipped with a Megaview II camera for digital image acquisition.

\section{Results}

\section{Characterization of chiNPs}

Chitosan chloridrate was successfully labelled with FITC as demonstrated by fluorescence analyses: the weight fraction of FITC per weight unit of chitosan was $7.7 \%$ (w/w). The FITC-chitosan conjugate was successfully transformed by ionotropic gelation with TPP into NPs with a spherical shape: mean diameter $158 \pm 17.8$ (P.I. 0.301) nm; Zeta-potential $12.7 \pm 0.8 \mathrm{mV}$, indicating a fair stability.

\section{Cell viability}

The Trypan blue exclusion test showed that the percentage of dead cells ranged from $0.13 \pm 0.10$ to $0.48 \pm 0.32$ in HeLa samples at all the retrieval times, at no variance with control samples not exposed to chiNPs $(0.37 \pm 0.27 \%)$.

\section{Intracellular distribution of chiNPs}

Fluorescence microscopy revealed similar intracellular distribution of chiNPs from $24 \mathrm{~h}$ to 14 days after NP retrieval. ChiNPs were found throughout cytoplasm in both interphasic and mitotic cells (Figure 1a) as well as in the nucleus or within the chromosomes cluster of mitotic cells (Figure $1 \mathrm{a}, \mathrm{b}$ ). Confocal microscopy showed that the fluorescence of FITC-labelled chiNPs may actually co-locate with the fluorescence signal of DNA in nuclear chromatin of interphase cells (Figure $1 \mathrm{c}, \mathrm{d}$ ). The mean percentage \pm SE of cells containing at least one chiNP varied from $4.77 \pm 0.74$ to $10.36 \pm 3.82$, with no significant difference among samples at different retrieval times. Up to 72h, NP-containing cells were randomly distributed in the whole population. However, at longer retrieval times (7 and 14 days), cells containing chiNPs formed discrete groups (Figure 2a). The fluorescence signals of chiNPs and lysosomes rarely co-distributed at any retrieval time indicating that most of the internalized chiNPs escaped lysosomal degradation (Figure 2 b,c). At transmission electron microscopy, chiNPs appeared as roundish and moderately electron-dense structures unequivocally labelled with the fine electron dense granular reaction product of DAB photo-oxidation (Figure 3). DAB precipitates were tiny and showed a weaker contrast than those previously observed in freshly internalised chiNPs; ${ }^{12}$ however, control samples confirmed the reliability of the reaction (Figure $3 \mathrm{f}, \mathrm{g}$ ). At all retrieval times, free-ranging chiNPs were found in the cytosol (Figure 3a); these were ubiquitously distributed in the cytoplasm, most occurring in the perinuclear region, but were never found in contact with any organelle. After $72 \mathrm{~h}$, and at 7 and 14 days, chiNPs were also observed inside dual-membrane-bounded autophagosomes (Figure $3 \mathrm{c}, \mathrm{d}$ ); chiNPs remnants still showing the photo-oxidation product were also found inside residual bodies (Figure $3 \mathrm{~d}, \mathrm{e}$ ). Consistent with the evidence at confocal microscopy, some chiNPS were also present inside the cell nucleus: these are always found in the nucleoplasm, often close to the nuclear envelope, and specific contact with particular nuclear domains has never been noticed (Figure 3b).

The ultrastructural analysis demonstrated no morphological alteration or damage of cell organelles in any sample.

\section{Discussion}

ChiNPs have been extensively studied as efficient drug-delivery carriers especially able to protect the encapsulated molecules from enzymatic degradation, thus enabling their long-lasting release in the intracellular
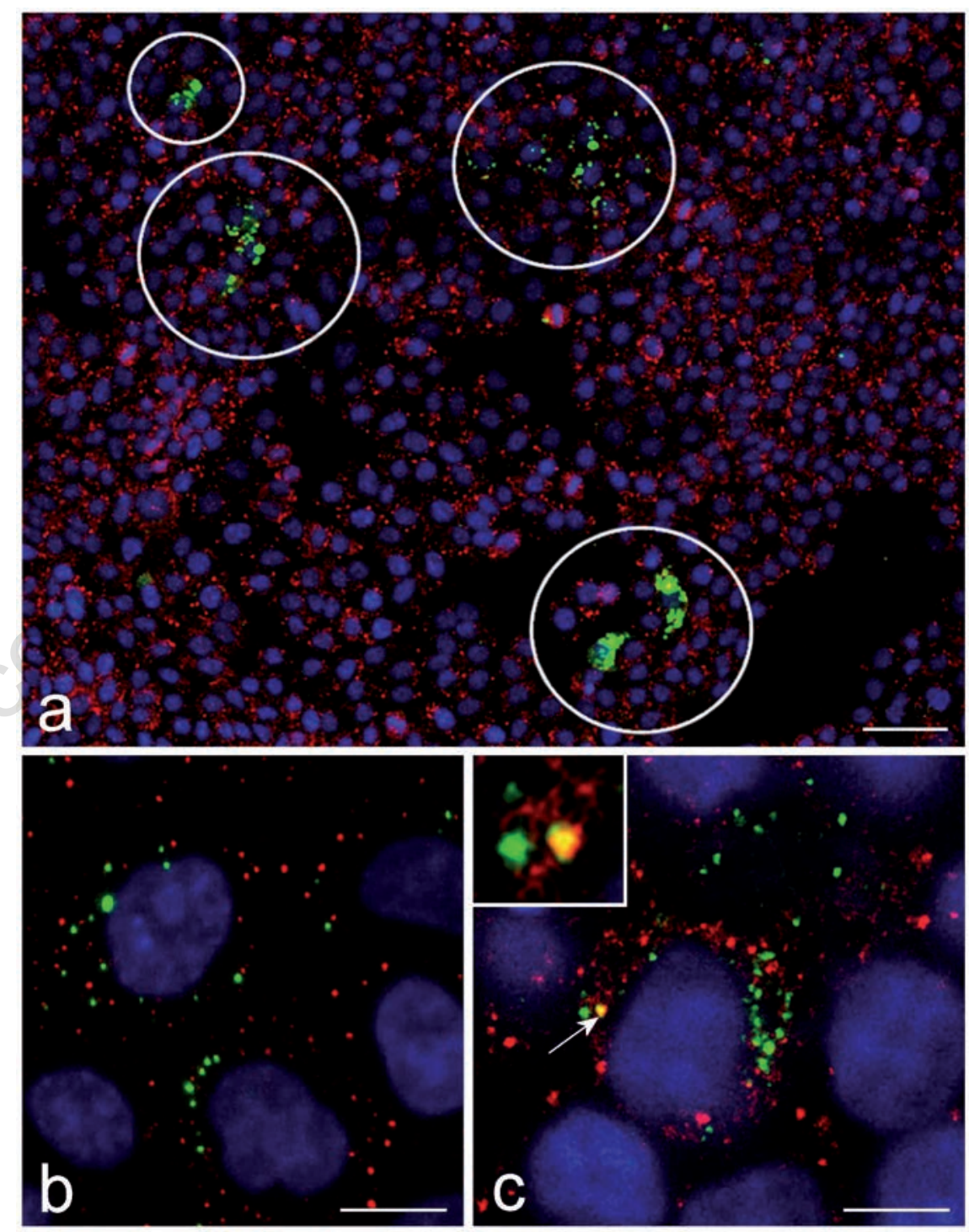

Figure 2. Confocal micrograph of HeLa cells 7 days after FITC-labelled chiNPs (green fluorescence) administration; immunolabelling for lysosomes (red fluorescence). a) Representative image of a HeLa cell culture: the white circles point to small cell clones containing chiNPs; scale bar: $200 \mu \mathrm{m}$. b, c) Most of the green and red fluorescence signals do not overlap, and only rarely (arrow and inset, in c) they colocalize. DNA was stained with Hoechst 33258 (blue fluorescence). Scale bars: $20 \mu \mathrm{m}$. 
milieu. ${ }^{10,13-16}$ However, the intracellular persistence and distribution of chiNPs in the long term has not been investigated yet.

In this work, correlative experiments using confocal fluorescence microscopy and electron microscopy after DAB photo-oxidation allowed to follow the fate of internalized chiNPs up to two weeks precisely describing their subcellular location, even when their morphology was hardly recognizable. ${ }^{20,21}$ The main result of the present study is the demonstration that internalized chiNPs persist inside the cell up to two weeks, occurring in both the cytoplasm and nucleus; accordingly, chiNPs are able to pass from mother to daughter cells through several mitotic cycles. In a previous study, internalized chiNPs were monitored from a few minutes to
$24 \mathrm{~h},{ }^{12}$ as well as after $24 \mathrm{~h}$ after retrieval. ${ }^{16}$ It was shown that chiNPs enter the cell via endocytosis and can thus be found inside endosomes early after internalization; however, many chiNPs escape endosomes and are found free in the cytosol by $24 \mathrm{~h}$. Accordingly, the amount of endosome-enclosed chiNPs decreases after retrieval, although a limited number persists up to $24 \mathrm{~h}^{16}$ This intracellular distribution and, in particular, the ability to escape the lysosomal pathway ensured an efficient drug release when these chiNPs were loaded with hypometabolising opioids. ${ }^{16}$ In the present work, we confirm and extend previous findings by showing that, up to 14 days, internalized chiNPs are mostly free in the cytosol and only rarely co-localize with lysosomes. The

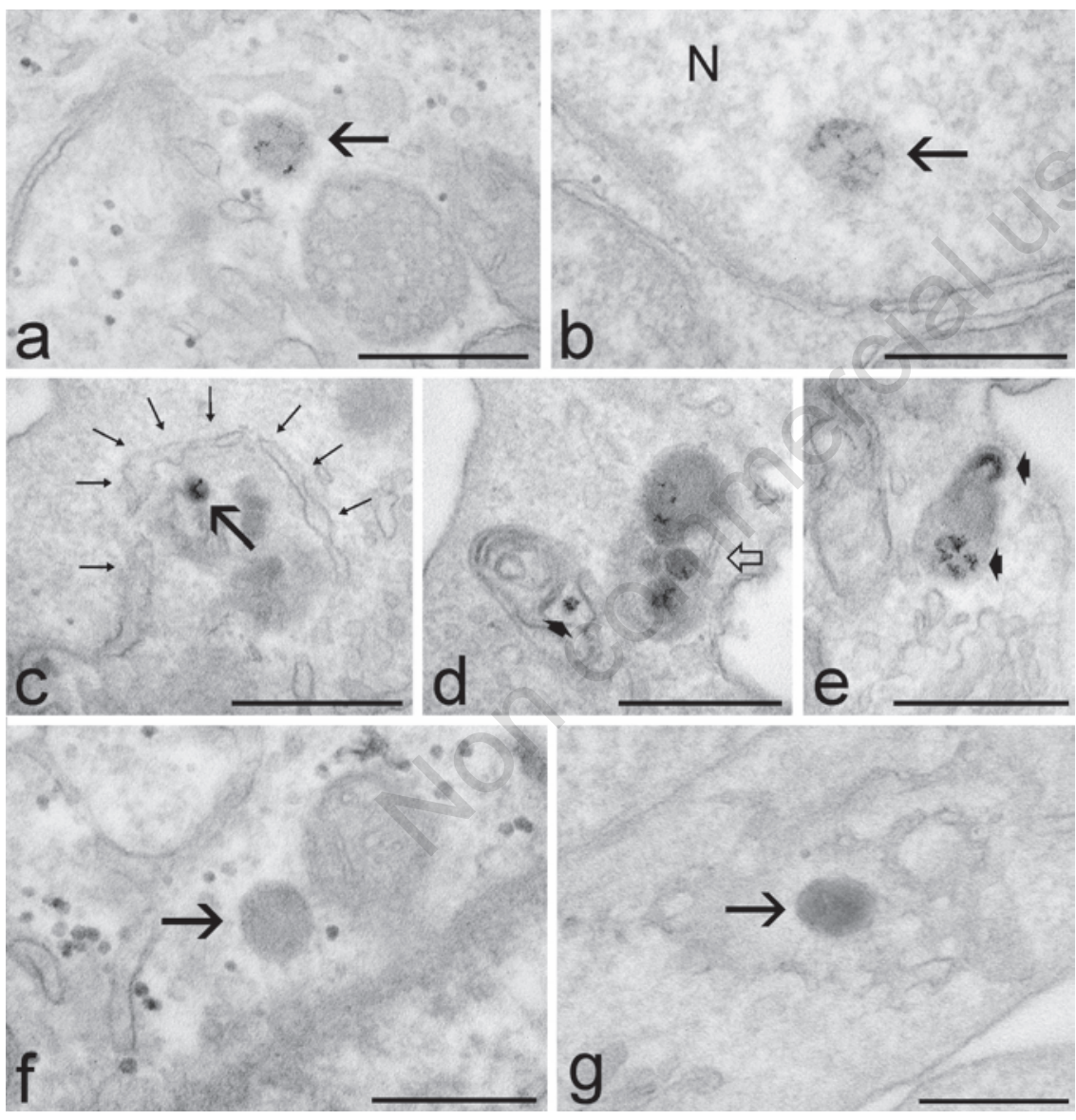

Figure 3. Transmission electron micrographs of HeLa cells $48 \mathrm{~h}(\mathrm{a}), 72 \mathrm{~h}(\mathrm{~b}, \mathrm{f}, \mathrm{g})$ and 7 days (c-e) after chiNP retrieval. The fine granular, dark reaction product of DAB photooxidation identifies chiNPs. a) A chiNP (arrow) occurs free in the cytoplasm. b) A chiNP (arrow) is located inside the cell nucleus $(\mathrm{N})$, close to the nuclear envelope. c) A dual membrane (thin arrows) partially surrounds a chiNP (arrow). d) A dual membrane autophagosome encircles three chiNPs (open arrow), and a residual body contains a chiNP remnant still showing the photo-oxidation product (arrowhead). e) A residual body contains chiNP remnants, recognizable by the dark reaction product (arrowheads). f,g) Control samples either exposed to the excitation light omitting DAB incubation (f) or incubated with DAB omitting light exposure (g). ChiNPs (arrows) do not show the fine granular, dark reaction product of DAB photo-oxidation. Scale bars: $500 \mathrm{~nm}$. specific mechanisms by which chiNPs escape endosomes are still unclear. However, similarly to other cationic polymers, this capability may be ascribed to a proton sponge effect: namely, certain cationic polymers can sequester the $\mathrm{H}^{+}$ ions whose increased concentration within the lumen of the endosome is responsible for the acidification taking place during the process of endo-lysosomal maturation; consequently, the influx of $\mathrm{Cl}^{-}$ions and water (which occurs in parallel with the proton influx to maintain electric neutrality) may cause osmotic swelling of the endosome up to its rupture point (reviewed in $^{9}$ ). Sustained cell viability and absence of organelle damage were observed in cells exposed to cationic-polymerbased NPs: this may be accounted for by either the inability of lysosomal enzymes to be active at the neutral $\mathrm{pH}$ of the cytosol, or by the rapid repair of the vesicle membrane after NP release preventing the efflux of lysosomal enzymes.

Thanks to the high resolution of transmission electron microscopy, we have here unequivocally demonstrated that, once free in the cytosol, chiNPs preserve their original morphology for long time and do not establish spatial relationships with any particular cell organelle or structure. The finding of dualmembrane structures surrounding cytoplasmic regions containing chiNPs, as well as the presence of residual bodies containing NP remnants suggest that NP degradation may anyway occur, probably via autophagy. However, the degradation of cytosolic chiNPs seems to be very slow, as demonstrated by their intracellular permanence up to two weeks after administration. Interestingly, the percentage of cells containing at least one chiNP does not significantly change during the experimental period, suggesting that the undegraded NPs are equally distributed to daughter cells, at mitosis. Consistently, the clusters of cells still containing chiNPs after 7 and 14 days post-administration apparently represent cell clones derived from a cell which massively internalized chiNPs.

According to previous findings, ${ }^{15}$ some chiNPs have been observed inside the cell nuclei. The size of the chiNPs used in both studies is incompatible with their passage through the nuclear pore complex; ${ }^{22,23}$ moreover, chiNPs have never been found inside nuclei at short times after internalization. ${ }^{12}$ It is likely that chiNPs do not enter the nucleus during interphase but are entrapped inside when the nuclear envelope reassembles at the end of mitosis, as previously supposed for other polymeric NPs. ${ }^{24}$ Similarly as it occurs in the cytoplasm, chiNPs do not make preferential contact with any nuclear domain.

In our experimental model, the cells did not show increased mortality or structural damage 
up to 14 day after chiNP exposure, but deeper investigation is mandatory on the functional effects that the presence of chiNPs in the nucleus may exert. Actually, long-time persistence of drug-loaded NPs in the nucleus may be considered as suitable whenever the release of a given agent must be sustained, but the question arises on the possible interference of this exogenous material per se with the overall nuclear functions, especially because of the polymer positive charges which could establish electrostatic interactions with the phosphate groups of nucleic acids. ${ }^{25}$

\section{References}

1. Kumar MNVR, Muzzarelli RAA, Muzzarelli C, Sashiwa H, Domb AJ. Chitosan chemistry and pharmaceutical perspectives. Chem Rev 2004;104:6017-84.

2. Hu L, Sun Y, Wu Y. Advances in chitosanbased drug delivery vehicles. Nanoscale 2013;5:3103-11.

3. El Kadib A, Bousmina M, Brunel D. Recent progress in chitosan bio-based soft nanomaterials. J Nanosci Nanotechnol 2014;14: 308-31.

4. Luo Y, Wang Q. Recent development of chitosan-based polyelectrolyte complexes with natural polysaccharides for drug delivery. Int J Biol Macromol 2014;64:353-67.

5. Schipper NGM, Olsson S, Hoogstraate JA, deBoer AG, Varum KM, Artursson P. Chitosans as absorption enhancers for poorly absorbable drugs 2 : mechanism of absorption enhancement. Pharm Res 1997;14:923-9.

6. Peppas NA, Huang Y. Nanoscale technology of mucoadhesive interactions. Adv Drug Deliv Rev 2004;56:1675-87.

7. Huang M, Ma Z, Khor E, Lim LY. Uptake of FITC-chitosan nanoparticles by A549 cells. Pharm Res 2002;19:1488-94.

8. Ma Z, Lim LY. Uptake of chitosan and asso- ciated insulin in Caco-2 cell monolayers: a comparison between chitosan molecules and chitosan nanoparticles. Pharm Res 2003;20:1812-9.

9. Varkouhi AK, Scholte M, Storm G, Haisma HJ. Endosomal escape pathways for delivery of biologicals. J Control Release 2011;151:220-8.

10. Wang JJ, Zeng ZW, Xiao RZ, Xie T, Zhou GL, Zhan XR, et al. Recent advances of chitosan nanoparticles as drug carriers. Int $\mathrm{J}$ Nanomedicine 2011;6:765-74.

11. Zaki NM, Nasti A, Tirelli N. Nanocarriers for cytoplasmic delivery: cellular uptake and intracellular fate of chitosan and hyaluronic acid-coated chitosan nanoparticles in a phagocytic cell model. Macromol Biosci 2011;11:1747-60.

12. Malatesta M, Giagnacovo M, Costanzo M, Conti B, Genta I, Dorati R, et al. Diaminobenzidine photoconversion is a suitable tool for tracking the intracellular location of fluorescently labelled nanoparticles at transmission electron microscopy. Eur J Histochem 2012;56:e20.

13. Sato T, Ishii T, Okahata Y. In vitro gene delivery mediated by chitosan. effect of $\mathrm{pH}$, serum, and molecular mass of chitosan on the transfection efficiency. Biomaterials 2001;22:2075-80.

14. Douglas KL, Piccirillo CA, Tabrizian M. Cell line-dependent internalization pathways and intracellular trafficking determine transfection efficiency of nanoparticle vectors. Eur J Pharm Biopharm 2008;68:676-87.

15. Colonna C, Dorati R, Conti B, Modena T, Biggiogera M, Spedito A, et al. Induction of an in vitro reversible hypometabolism through chitosan-based nanoparticles. J Microencapsul 2011;28:229-39.

16. Malatesta M, Galimberti V, Cisterna B, Costanzo M, Biggiogera M, Zancanaro C. Chitosan nanoparticles are efficient carriers for delivering biodegradable drugs to neuronal cells. Histochem Cell Biol
2013;141:551-8.

17. Tang ES, Huang M, Lim LY. Ultrasonication of chitosan and chitosan nanoparticles. Int J Pharm 2003;265:103-14.

18. Colonna C, Conti B, Perugini P, Pavanetto F, Modena T, Dorati R, et al. Chitosan glutamate nanoparticles for protein delivery: development and effect on prolidase stability. J Microencapsul 2007;24:553-64.

19. Alpini C, Lotzniker M, Valaperta S, Bottone MG, Malatesta M, Montanelli A, et al. Characterization for anti-cytoplasmic antibodies specificity by morphological and molecular techniques. Autoimmunity Highlights 2012;3:79-85.

20. Malatesta M, Zancanaro C, Costanzo M, Cisterna B, Pellicciari C. Simultaneous ultrastructural analysis of fluorochromephotoconverted diaminobenzidine and gold immunolabelling in cultured cells. Eur J Histochem 2013;57:e26.

21. Malatesta M, Pellicciari C, Cisterna B, Costanzo M, Galimberti V, Biggiogera M, et al. Tracing nanoparticles and photosensitizing molecules at transmission electron microscopy by diaminobenzidine photooxidation. Micron 2014;59C:44-51.

22. Allen TD, Cronshaw JM, Bagley S, Kiseleva E, Goldberg MW. The nuclear pore complex: mediator of translocation between nucleus and cytoplasm. J Cell Sci 2000; 3113:1651-9.

23. Labokha AA, Fassati A. Viruses challenge selectivity barrier of nuclear pores. Viruses 2013;5:2410-23.

24. Guan M, Zhu Q, Liu Y, Bei Y-Y, Gu Z-L, Zhang X-N, et al. Uptake and transport of a novel anticancer drug-delivery system: lactosyl-norcantharidin-associated N-trimethyl chitosan nanoparticles across intestinal Caco-2 cell monolayers. Int $\mathrm{J}$ Nanomedicine 2012;7:1921-30.

25. Lai WF, Lin MC. Nucleic acid delivery with chitosan and its derivatives. J Control Release 2009;134:158-68. 